\title{
Relation of measured brain glucose utilisation and cerebral atrophy in man
}

\author{
NICHOLAS L SCHLAGETER,* BARRY HORWITZ,* HELEN CREASEY, *§ \\ RICHARD CARSON, $\dagger$ RANJAN DUARA, ${ }^{*} \ddagger$ GARY W BERG,$*$ \\ STANLEY I RAPOPORT* \\ From the Laboratory of Neurosciences, National Institute on Aging, ${ }^{*}$ National Institutes of Health, and the \\ Department of Nuclear Medicine, Clinical Center, $\dagger$ National Institutes of Health, Bethesda, MD, USA
}

SUMMARY The effect of cerebral atrophy on measured cerebral metabolic rates for glucose (CMRglc), as determined with positron emission tomography (PET), was examined in 49 healthy males aged 21-83 years. Global CMRglc and regional CMRglc for 34 grey matter regions parallel to and from 30 to $80 \mathrm{~mm}$ above the inferior orbital meatal (IOM) line were measured under resting conditions, using [18F]-fluorodeoxyglucose and an ECAT II positron emission tomograph. Using a GE $8800 \mathrm{CT} / \mathrm{T}$ scanner, slices parallel to and from 30 to $80 \mathrm{~mm}$ above the IOM line were analysed for CSF volume. Cerebral atrophy, indicated by increased CSF volume, was correlated significantly with global CMRglc, but accounted for no more than $13 \%$ of the variance in the CMRglc measurements. Methods for correcting for inter-subject variation in CSF volume were proposed. Global values for CMRglc, uncorrected or corrected for CSF volume, were found to be age invariant. These findings indicate that $(a)$ cerebral atrophy has a small, but statistically significant effect on CMRglc as measured with PET; $(b)$ CMRglc is age invariant in healthy males.

Positron emission tomography (PET) has been used in several centres to study the relation between aging and brain oxidative metabolism, but the reported results have been discrepant. The cerebral metabolic rate for oxygen $\left(\mathrm{CMRO}_{2}\right)$, determined with PET using ${ }^{15} \mathrm{O}$, was found not to decline with age in studies done by Frackowiak et al, ${ }^{12}$ and by Pantano et $a l^{3}$ The cerebral metabolic rate for glucose (CMRglc), as estimated by PET using $\left[{ }^{18} \mathrm{~F}\right]-2-$ fluoro-2-deoxy-D-glucose (FDG), was found by Kuhl et $a l^{4}$ to decline with age. In contrast, deLeon et $a l^{5}$ found no age-related decline in global or regional cerebral glucose metabolism, in agreement with prior studies from our laboratory. ${ }^{7}$ The above investigations were done on healthy volunteers, and PET scanning was performed under experimental

†Present address: Department of Nuclear Medicine, Mount Sinai Medical Center, 4300 Alton Road, Miami Beach, FL 33140, USA. $\S$ Present address: Lidcombe Hospital, Joseph St, Lidcombe, New South Wales, Australia.

Address for reprint requests: B Horwitz, Building 10, Room $6 \mathrm{C103}$, NIH, Bethesda, MD 20892, USA

Received 31 December 1985 and in revised form 30 April 1986 Accepted 15 May 1986 conditions which differed with respect to the uptake period, the amount and type of sensory stimulation, and the PET machine used.

Because of the limited resolution of PET scanners in use today, cerebral atrophy, consistently demonstrated to accompany healthy aging, ${ }^{59}$ may influence the measured CMRglc. Global measures of glucose metabolism of the brain, which include variable proportions of grey matter, white matter, and metabolically inactive cerebrospinal fluid (CSF), may be affected by differences in cerebral atrophy in comparing young and old subjects. Some investigators have proposed, therefore, that the measured CMRglc be corrected for CSF volume, using measures of cerebral atrophy derived with computed transverse axial tomography (CT). ${ }^{1011}$

The present study reports our findings of CMRglc and brain morphometric data, as obtained by quantitative $C T{ }^{8}$ on 49 healthy male subjects ranging in age from 21 to 83 years. These data were analysed to determine if CSF volume was correlated with CMRglc. We found that hemispheric metabolism (hCMRglc) and average weighted grey matter metabolism (gCMRglc) were significantly related (inversely) to CSF volume. A method for correcting 
hCMRglc and gCMRglc, based on this relation, is proposed. After the correction, hemispheric metabolism (hCMRglc) and average weighted grey matter metabolism (gCMRglc) remained age invariant. An abstract of part of this work has been published. ${ }^{12}$

\section{Materials and methods}

\section{Subject selection}

Screening of male volunteers was done with a complete history and physical examination, chest radiography, electrocardiogram, and blood tests of haematological, metabolic and endocrine functions, as described by Duara et al $^{6{ }^{7}} \mathrm{Sub}-$ jects were excluded if they had a history of head injury, drug or alcohol abuse, a major psychiatric disorder, or had evidence of cardiovascular, cerebrovascular, or neurosensory disorder. Sixty healthy males between the ages of 21 to 83 years were thus recruited. All subjects signed an informed consent that described the purpose and procedure of the study as well as the risks involved.

Each volunteer had his CT and PET scans at least 3 months apart (longest interval 1 year). Owing to death (2 subjects) and to failure to return for one scan or the other ( 9 subjects), only 49 volunteers underwent both PET and CT scans. Table 1 contains physiological data on these subjects.

Table 1 Characteristics of the subjects

Variable

No. of subjects

Age (yr)

Weight $(\mathrm{kg})$

Height (cm)

Heart rate (beats/min)

Systolic blood pressure $(\mathrm{mm} \mathrm{Hg})$

Diastolic blood pressure $(\mathrm{mm} \mathrm{Hg})$

Plasma glucose ( $\mathrm{mg} \%)$

Arterial oxygen tension $(\mathrm{mm} \mathrm{Hg})$

Arterial $\mathrm{CO}_{2}$ tension $(\mathrm{mm} \mathrm{Hg}$ )

Arterial pH

\begin{tabular}{cc}
49 & \\
51 & \pm 19 \\
76 & \pm 9 \\
177 & \pm 7 \\
68 & \pm 13 \\
124 & \pm 14 \\
76 & \pm 10 \\
86 & \pm 9 \\
83 & \pm 16 \\
38 & \pm 8 \\
$7.40 \pm 0.02$ \\
\hline
\end{tabular}

Mean \pm 1 standard deviation are given.

\section{PET scanning}

Details of our PET scanning procedure have been described. ${ }^{6}{ }^{18} \mathrm{~F}$-2-deoxyglucose-D-glucose (FDG) was synthesised using ${ }^{18} \mathrm{~F}$-labelled hypofluorite, as described by Shiue et al. ${ }^{13}$ The subject was placed in a supine position with his ears plugged with cotton and was blindfolded; ambient light was reduced. After a 30 minute acclimatisation period, 3 to $5 \mathrm{mCi}$ of FDG was injected intravenously. From the time of injection until the end of scanning, serial samples of "árterialised" venous blood or of arterial blood were obtained via an indwelling arterial or venous catheter for analysis of plasma glucose and radioactivity. Starting 45 minutes after injection, PET scanning was performed with an ECAT II tomograph (Ortec, Oak Ridge, TN) in the medium resolution mode (full width at half maximum, 1.7 $\times 1.7 \mathrm{~cm}$ ). Interslice distance was $1.4 \mathrm{~cm}$ (centre to centre). Up to seven serial images were obtained in a plane parallel to and 5 to $100 \mathrm{~mm}$ above the externally defined inferior orbital meatal (IOM) line.

Reconstruction of PET images was performed with the
ORTEC supplied attenuation correction program using a uniform attenuation coefficient $\left(\mu=0.088 \mathrm{~cm}^{-1}\right)$ and an operator drawn ellipse to define the skull edge.

\section{CT scanning}

CT scans were performed, without contrast enhancement, with a GE 8800 CT/T (General Electric, Milwaukee, WI) scanner (full width at half maximum $=1 \mathrm{~mm}$ ). Serial slices were obtained in a plane parallel to the IOM line. Each slice was $10 \mathrm{~mm}$ thick and was separated from adjacent slices by $7 \mathrm{~mm}$.

\section{Data analysis}

Analysis of both PET and CT images started with a comparison of each slice with anatomical sections from an atlas of a human brain. ${ }^{14}$ The level above the IOM line as given in the atlas was assigned to each slice. "Mm above atlas IOM line", therefore, is a reference value allowing comparison of equivalent slices between subjects.

PET scans were selected between 30 and $80 \mathrm{~mm}$ above the IOM line. As described by Duara et al, ${ }^{6}$ hemispheric regions of interest (ROI) were outlined, as were 16 bilaterally symmetrical and two midline ROI. Figure 1 shows three representative PET and CT slices with ROI outlined on the PET scans. Calculation of hemispheric CMRglc and of regional CMRglc (rCMRglc) in each image was done with a four rate-constant operational equation, using grey matter transfer constants (see Duara et $a l^{6}$ for values) and a "lumped constant" of $0.418 .{ }^{15} \mathrm{CMRglc}$ was expressed in units of $\mathrm{mg}$ glucose $/ 100 \mathrm{~g}$ tissue/min. If an anatomical region appearedo on more than one slice, a weighted mean rCMRglc was cal $\Omega$ culated as follows:

$$
\text { rCMRglc }=\frac{\Sigma_{\mathrm{i}}(\mathrm{rCMRglc})_{\mathrm{i}} \mathrm{N}_{\mathrm{i}}}{\Sigma_{\mathrm{i}} \mathrm{N}_{\mathrm{i}}}
$$

where $\mathbf{N}_{\mathrm{i}}$ equals the number of pixels in the ROI in slice $i$ and $\mathrm{i}=1,2 \ldots$ number of slices in which the ROI appears. Weighted grey matter CMRglc (gCMRglc) was derived by substituting all rCMRglc from grey matter regions into equation 1, whereas weighted hemispheric CMRgic (hCMRglc) was obtained by using the right and left hemispheric CMRglc obtained from all slices between 30 and $80 \mathrm{~mm}$ above the IOM line.

CT scans between 30 to $80 \mathrm{~mm}$ above the IOM line were analysed in two ways, as described by Schwartz et $a l^{8}$ and DeLeo et al. ${ }^{16}$ First, a digital analysis system was used to measure the total number of pixels (surface area elements) of grey matter, white matter, and CSF in each slice. A second procedure utilised an edge-finding program, which located the inner edge of the skull, to determine intracranial cross sectional area in a given slice. Volumes of the above structures were calculated using the formula:

$$
V=0.00448 \Sigma_{i} N_{i}
$$

where $i$ sums over the number of slices containing an ROI of an identified structure, $N_{i}$ equals the number of pixels in the $\mathrm{ROI}$ of a slice, and 0.00448 represents the product of cross sectional area per pixel $\left(0.0064 \mathrm{~cm}^{2}\right)$ and interslice distance $(0.7 \mathrm{~cm})$. Thus, data on intracranial volume (ICV), and CSF volume (CSFV) were obtained from each scan. Note that these volumes represent values only between 30 to $80 \mathrm{~mm}$ above the IOM line and not the total volumes within the skull. The analysis was limited to these slices because of 
important beam hardening artifacts in CT scans below $30 \mathrm{~mm}$ or above $80 \mathrm{~mm}$ above the IOM line. ${ }^{817}$

Statistical analysis

Pearson product moment correlation coefficients were used to examine relations between variables, regression equations were calculated by the method of least squares, and $t$ tests were used to compare means. ${ }^{18}$ Statistical significance was taken as $\mathrm{p}<0.05$.

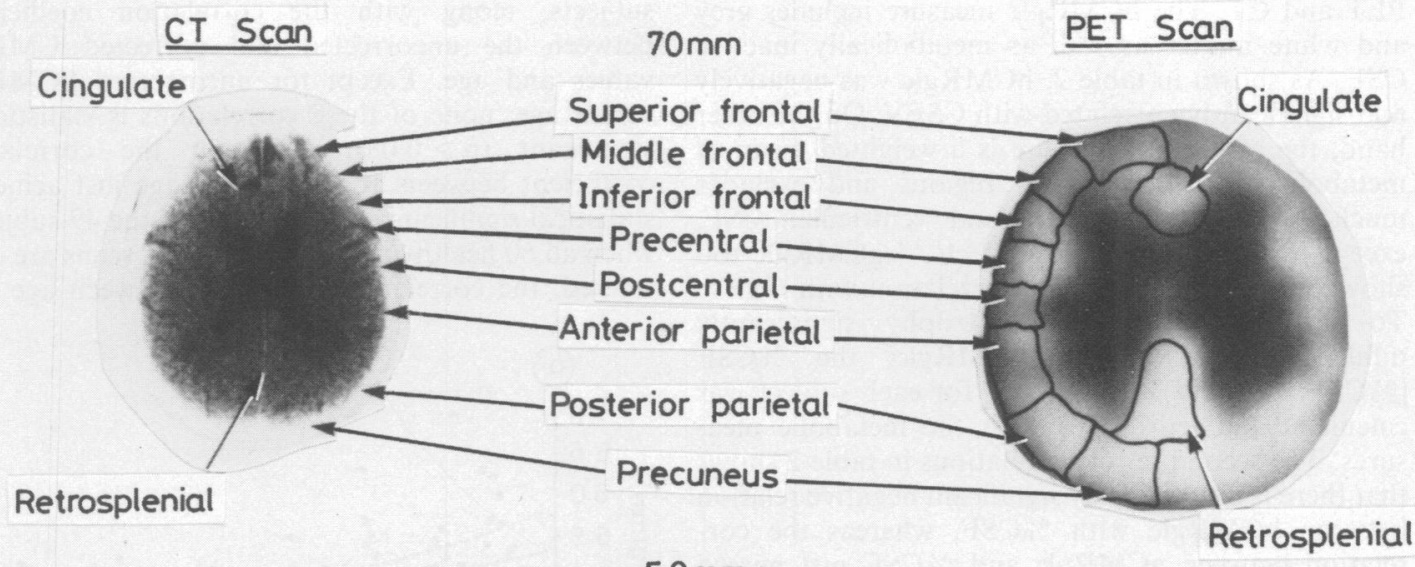

$50 \mathrm{~mm}$
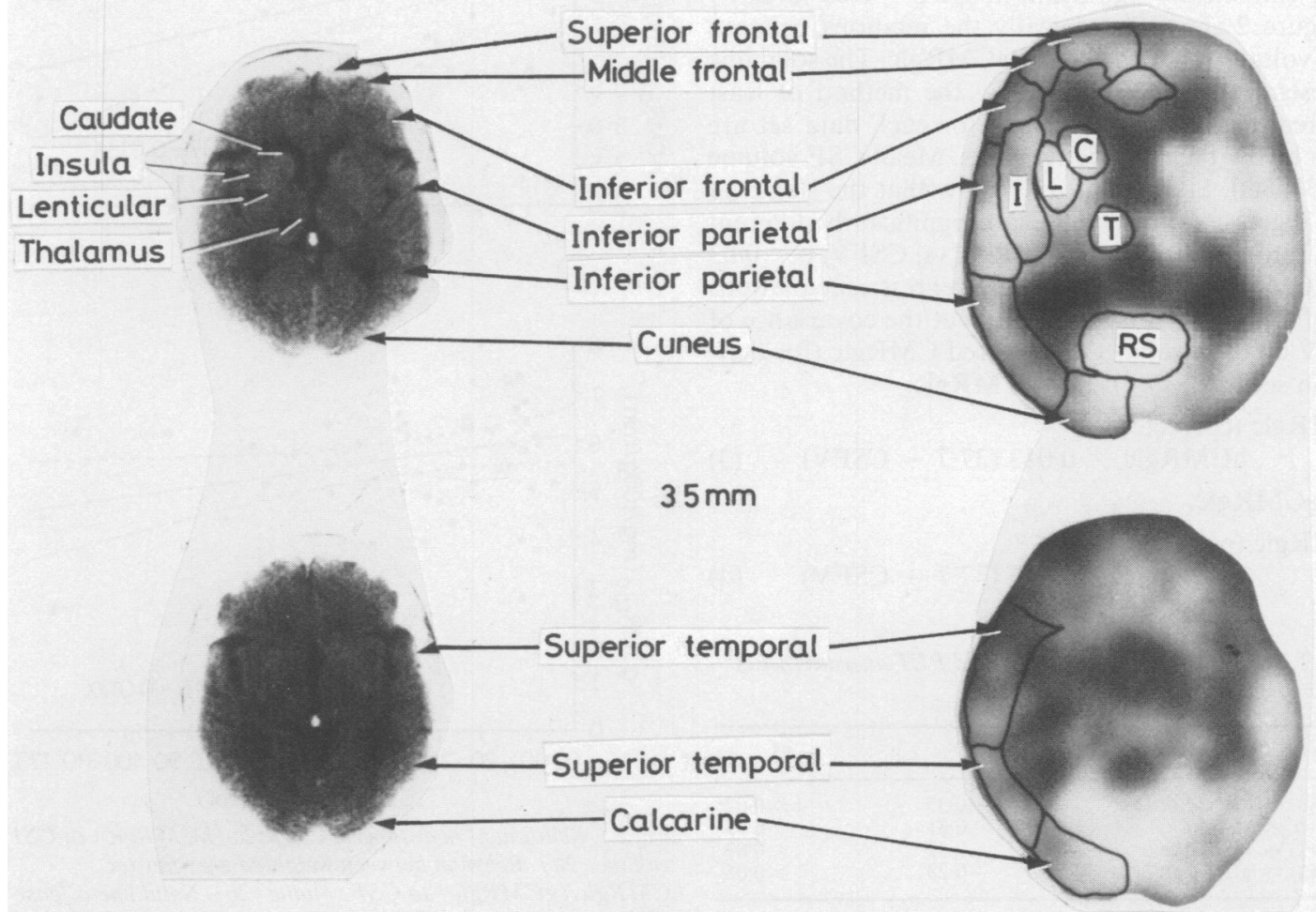

Fig $1 C T$ and PET scan slices from a 72 year old man at 70,50, and $35 \mathrm{~mm}$ above the inferior orbital meatal (IOM) line as defined in the atlas of Eycleshymer and Schoemaker. ${ }^{14}$ Regions of interest are outlined on the PET scan slices. Abbreviations: $C$, head of caudate nucleus; $L$, lenticular nucleus; $I$, insula; $T$, thalamus; $R S$, retrosplenial region. 


\section{Results}

Table 2 lists Pearson product moment correlation coefficients between CT determined intracranial volumetric measures, and metabolic rates as measured with PET, in the 49 subjects who underwent both PET and CT. The hCMRglc measure includes grey and white matter as well as metabolically inactive CSF. As shown in table 2, hCMRglc was negatively and significantly correlated with CSFV. On the other hand, the gCMRglc measure is a weighted mean of metabolism in grey matter regions and excludes much of the white matter and ventricular CSF, except for partial voluming effects. ${ }^{19}$ gCMRglc too showed a significant inverse correlation with CSFV. To determine if cerebral atrophy significantly influences hCMRglc or gCMRglc, the \%CSF $[\% \mathrm{CSF}=(\mathrm{CSFV} / \mathrm{ICV}) \times 100]$ for each subject was calculated and correlated with the metabolic measures. The second set of correlations in table 2 shows that there is a statistically significant negative relation between hCMRglc with \%CSF, whereas the correlation between gCMRglc and \%CSF just missed being statistically significant at the $\mathrm{p}<0.05$ level.

Figure 2 shows graphically the relations between CSF volume, hCMRglc, and gCMRglc. The solid line represents the best data fit, by the method of least squares. Regression equations for each data set are given below their respective plots. Mean CSF volume was $37.7 \mathrm{ml}$. Student's $t$ tests show that the slopes of both regression lines in fig 2 are significantly different from zero ( $p<0.02$ for hCMRglc vs. CSFV; $p<0.03$ for gCMRglc vs. CSFV). These regression equations therefore can be used to partial out the covariance of CSFV on each subject's measured CMRglc (for derivation see Appendix): For hCMRglc:

hCMRglc (corrected) $=$

$$
\text { hCMRglc }-0.013(37.7-\text { CSFV) }
$$

For gCMRglc:

gCMRglc (corrected) $=$

$$
\text { gCMRglc }-0.013(37 \cdot 7-\text { CSFV) }
$$

Table 2 Correlations between CT and PET measurements in 49 men

\begin{tabular}{lll}
\hline Variables & $r^{*}$ & $p \dagger$ \\
\hline CSFV vs. hCMRglc & -0.35 & $<0.02$ \\
CSFV vs. gCMRglc & -0.31 & $<0.03$ \\
\%CSF( ) vs. hCMRglc & -0.32 & $<0.03$ \\
$\%$ CSF $(\ddagger)$ vs. gCMRglc & -0.28 & $\geqslant 0.05$ \\
\hline
\end{tabular}

*Pearson product moment correlation coefficients.

†Significance level probability.

$+\%$ CSF $=($ CSFV $/$ ICV $) \times 100$

Abbreviations: ICV, Intracranial volume between 30 and $80 \mathrm{~mm}$ above the IOM line; CSFV, Cerebrospinal fluid volume; hCMRglc and $\mathrm{gCMRglc}$ are as given in the text.
Correction of each subject's hCMRglc and gCMRglc, using Eqs. (3) and (4), was performed and the corrected values were examined for their correlation with age. Table 3 gives the values for mean hCMRglc $( \pm S D)$ and mean gCMRglc $( \pm S D)$, uncorrected and corrected for CSF volume, for the 49 subjects, along with the correlation coefficients between the uncorrected and corrected CMRglc values and age. Except for uncorrected hCMRglc versus age, none of these correlations is statistically significant $(p>0.05)$. Although the correlation coefficient between hCMRglc and age just achieved statistical significance $(p=0.045)$ for the 49 subjects, when all 60 healthy men who had PET scans are considered, the correlation coefficient between age and
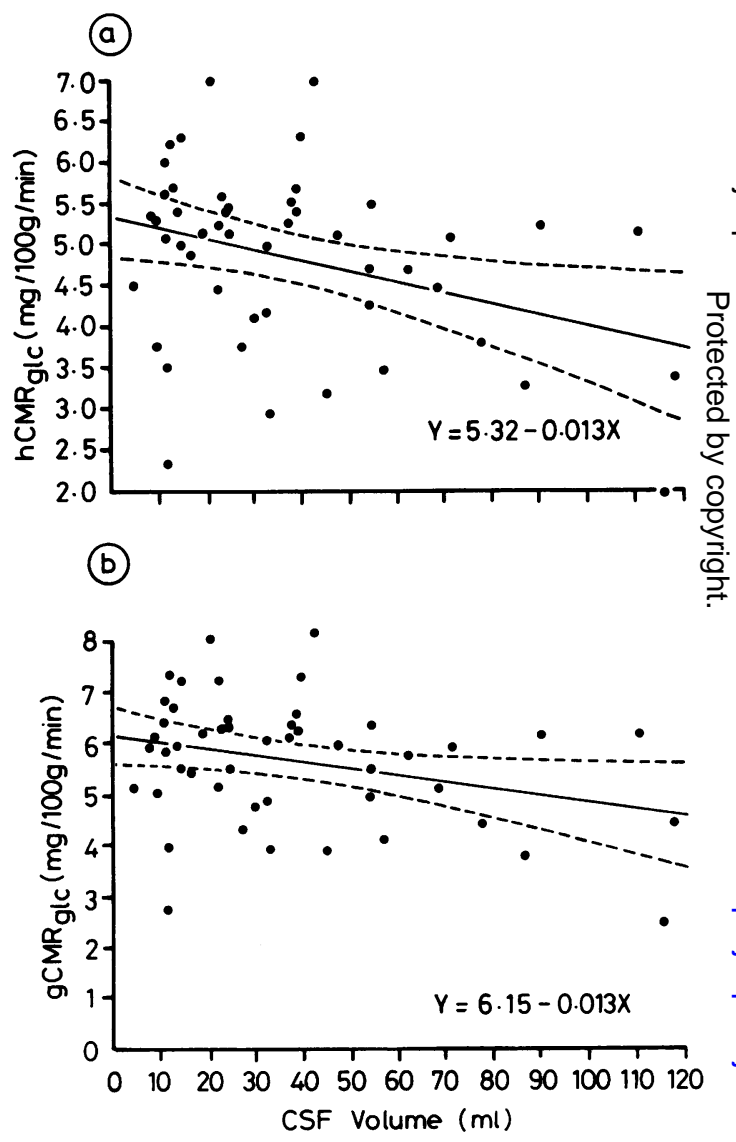

Fig 2 Relation of hemispheric CMRglc (hCMRglc) to CSF volume (2a). Relation between weighted grey matter

CMRglc ( $\mathrm{gCMRglc}$ ) to CSF volume (2b). Solid line is least squares fit and dashed lines are $95 \%$ confidence intervals for mean predicted values. Metabolic rates are in units of mg glucose/100 g tissue/min. Regression equations are given below their respective plots. The equation format is $y=b+m x$. 
hCMRglc loses statistical significance $(r=-0 \cdot 21$, $\mathrm{p}=0 \cdot 10)$.

Another way to examine the effect CSFV has on the relation between cerebral metabolism and age is by using partial correlation coefficients. ${ }^{18}$ Partial correlations between hCMRglc and age and between gCMRglc and age, in which the control variable is CSFV, are -0.06 and -0.04 , respectively. Neither of these is statistically significant ( $p>0.05)$.

A third way to examine how CSFV affects the agemetabolism relation is based on a correction technique proposed by Herscovitch et al. ${ }^{11}$ They compute a corrected hCMRglc that has been rescaled by the ratio of intracranial volume to metabolically active brain volume:

hCMRglc(r) $=$ hCMRglc [ICV/(ICV-CSFV)]

where hCMRglc(r) is the corrected (rescaled) hCMRglc. The last entry in table 3 gives the mean hCMRglc(r) for the 49 men, along with the correlation between $\mathrm{hCMRglc}(\mathrm{r})$ and age. As with the preceding two procedures, the result is not statistically significant $(p>0.05)$. The percent increase in hCMRglc due to rescaling averaged $5.0 \%$ (SD 3.9\%), and was significantly correlated with age $(r=0.71$, $\mathrm{p}<0.0001)$.

Table 3 Means and correlations between PET measurements and age in 49 men

\begin{tabular}{llll}
\hline Variables & Mean $\pm S D^{*}(1)$ & $r \dagger$ & $p \ddagger$ \\
\hline hCMRglc & $4.83 \pm 1.09$ & -0.29 & $0.045 \S$ \\
gCMRglc & $5.66 \pm 1.02$ & -0.25 & 0.09 \\
hCMRglc(c) & $4.83 \pm 1.02$ & -0.04 & 0.76 \\
gCMRglc(c) & $5.66 \pm 1.16$ & -0.03 & 0.84 \\
hCMRglc(r) & $5.06 \pm 1.11$ & -0.18 & 0.20 \\
\hline
\end{tabular}

*Units: $\mathrm{mg}$ glucose $/ 100 \mathrm{~g}$ tissue/min.

+Pearson product moment correlation coefficients.

tSignificance level probability.

\&Statistically significant.

Abbreviations: hCMRglc and gCMRglc are as given in the text; hCMRglc(c) and gCMRglc(c) are the PET measurements corrected for CSF volume, given by Eqs. (3) and (4), respectively. hCMRglc(r) is the rescaled hCMRglc, given by Eq. (5).

\section{Discussion}

The PET and CT correlations listed in table 2 show that CSF volume is significantly and negatively correlated with gCMRglc and hCMRglc. Because hCMRglc includes sulcal and ventricular CSF, which is metabolically inert, the finding that CSF volume and hCMRglc are inversely related is not unexpected. The gCMRglc measure, however, excludes much of the white matter as well as CSF. The negative correlation of this parameter with CSF volume indicates that, because of the limited resolution of our PET scanner, a significant amount of CSF is included in
rCMRglc calculations and that the partial voluming from CSF in grey matter regions is marked. ${ }^{19}$ That $\% \mathrm{CSF}$ is significantly and negatively correlated with hCMRglc supports the hypothesis that cerebral atrophy influences metabolic rate measurements, although the influence is small. Note that only $10 \%$ of the variance in hCMRglc can be accounted for by \%CSF; for gCMRglc, which just missed being statistically correlated with $\% \mathrm{CSF}$, less than $8 \%$ of the PET measure is accounted for by \%CSF.

Corrections for the effect of CSF volume on hCMRglc and gCMRglc were performed in three different ways. First, we plotted these data, as shown in fig 2 , and then derived regression equations. To partial out the effect of inter-subject differences in CSF volume on cerebral metabolism, the regression equations were modified [Eqs. (3) and (4)] and a corrected metabolic rate was calculated for each of the 49 subjects. These corrected values (for either hCMRglc or gCMRglc) allow us to compare brain metabolism as if each subject had the same CSFV. Second, we used the method of partial correlation coefficients to examine directly the relation between PET measurements and age, with CSFV as the controlled variable. Finally, we used the rescaling approach of Herscovitch $e t a^{11}$ to obtain corrected values for hCMRglc. All three methods show clearly that cerebral metabolism, when corrected for cerebral atrophy, is age invariant.

Herscovitch et $a l^{11}$ found that, owing to CSF, global cerebral blood flow and $\mathrm{CMRO}_{2}$ were underestimated by an average of $5 \%$ in a group of 10 young normal subjects (mean age: $27.5 \mathrm{yrs}$ ), and by an average of $11 \%$ in a group of eight elderly controls (mean age: $72.8 \mathrm{yrs}$ ). Alavi et al ${ }^{10}$ examined 12 young (age range: $19-32$ yrs) and two elderly (48-54 yrs) controls using a rescaling method similar to that of Herscovitch et al. ${ }^{11}$ They found that global CMRglc was underestimated by an average of about $8 \%$ in both groups. The result of $5 \%$ that we obtain for our much larger sample of 49 men is comparable to values from these other studies.

Besides increasing the amount of metabolically inactive CSF, tissue atrophy can also affect PET measurements by changing the photon attenuation characteristics of the brain. Thus, as CSF (water) replaces brain tissue (whose specific gravity ${ }^{20}$ is 1.04), there is diminished attenuation. However, this effect is very small (the attenuation coefficient in water is $3 \%$ smaller than in brain ${ }^{21}$ ), and can be neglected even though our method for reconstructing PET images employs a constant photon attenuation coefficient.

This study, in agreement with previous reports from this laboratory, ${ }^{67}$ demonstrates that resting cerebral glucose utilisation, when measured under conditions of reduced sensory stimulation, is age 
invariant in our sample of healthy males between the ages of 21 and 83 years. Hemispheric CMRglc and weighted grey matter gCMRglc (for 60 men) show no statistically significant reduction with age, whether or not they are corrected for cerebral atrophy. The tendency for uncorrected CMRglc to decline with age, as indicated by a negative correlation coefficient that approaches statistical significance, is essentially eliminated when CMRglc is corrected for tissue atrophy.

Our findings agree with several previous reports, ${ }^{5-7}$ but differ from the results reported by Kuhl and associates, ${ }^{4}$ who found that CMRglc values decline in relation to age in a mixed population of males and females who were scanned with eyes open and ears unplugged. However, their values of CMRglc were not corrected for the effects of brain atrophy. Moreover, Duara et $a^{22}$ recently reported CMRglc to be significantly higher in young females compared to old females, but CMRglc did not differ significantly between young and old males. Thus, the age-associated decline in CMRglc reported by Kuhl et $a l^{4}$ may be due to sex differences rather than agerelated changes in brain metabolism, or to the effects

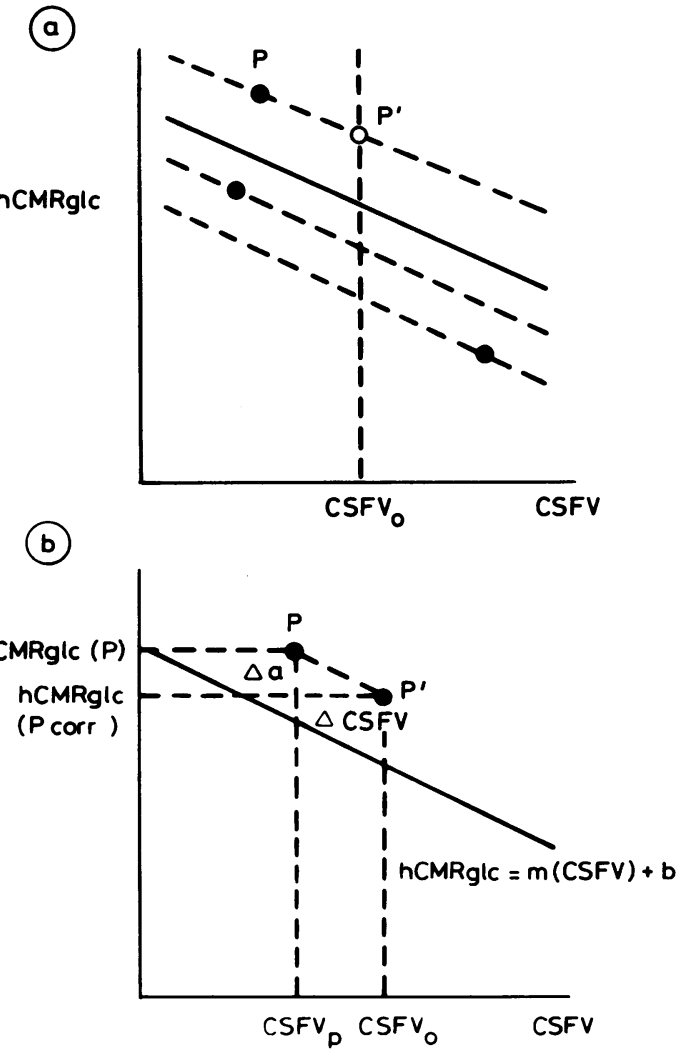

Fig 3 For explanation of $3 a$ and $3 b$, see text of Appendix. of brain atrophy, or to differences in subject selection, or to the presence of visual and auditory input, or to some combination of these factors.

Although the findings of our laboratory show that global and most regional values of CMRglc do not decline with age, ${ }^{67}$ changes in the pattern of cerebral metabolism do occur with aging in the same subjects. Horwitz et $a^{23}$ found that, compared with a young group, elderly subjects have fewer statistically significant interregional correlations of rCMRglc within the parietal lobe, and between parietal and frontal lobe regions.

In summary, this study quantifies the effect of cerebral atrophy on two measures of global cerebral metabolism. This effect, although statistically significant, accounts for only a small amount of the variance found when CMRglc is measured in human subjects. However, the methods proposed can be used to correct measured CMRglc in subjects with brain pathology. Patients with Alzheimer's disease, for example, have progressive cerebral atrophy when compared to age matched controls. ${ }^{24-26}$ Thus correction for CSF volume may be necessary.

\section{Appendix}

In this appendix, we show how Eqs. (3) and (4) are derived. Because both equations have the same form, we shall consider only Eq. (3).

The relation between hCMRglc and CSFV, shown in fig $2 a$, is illustrated, for a few subjects, in fig $3 a$ CSFVo $(37.7 \mathrm{ml})$ is the mean CSF volume of all 49 subjects in the study. The solid line is the regression line obtained by the method of least squares for these 49 subjects. The equation of the regression line is

$$
\text { hCMRglc }=m(C S F V)+b
$$

where $\mathrm{m}$ and $\mathrm{b}$ are the least square parameters $(\mathrm{m}=-0.013 \mathrm{mg}$ glucose $/ 100 \mathrm{~g} / \mathrm{min} / \mathrm{ml}, \mathrm{b}=5.32 \mathrm{mg}$ glucose $/ 100 \mathrm{~g} / \mathrm{min}$ ). Point $P$ represents one subject whose CSF volume has value CSFVp, and whose hCMRglc has value hCMRglc(P). The dashed lines, one for each subject, are parallel to the regression line.

The corrected metabolic values are obtained by normalising each subject's metabolic rate with respect to the mean CSF volume. This is done by moving each point, such as $\mathbf{P}$, along its dashed line parallel to the regression line, so that it intersects the vertical CSFVo line. Thus, as shown in fig $3 \mathrm{~b}$, the corrected hCMRglc corresponds to point $\mathbf{P}^{\prime}$. Because the regression line has slope $\mathrm{m}$, the geometry of fig $3 \mathrm{~b}$ implies

$$
\begin{aligned}
& \mathrm{m}= \Delta \mathrm{a} / \Delta(\mathrm{CSFV})= \\
& \frac{\mathrm{hCMRglc}(\mathrm{P})-\mathrm{hCMRglc}(\mathrm{P}, \text { corrected })}{\text { CSFVo }- \text { CSFVp }}
\end{aligned}
$$


or,

$$
\begin{aligned}
& \text { hCMRglc }(\mathrm{P}, \text { corrected })= \\
& \text { hCMRglc }(\mathrm{P})-\mathrm{m}(\mathrm{CSFVo}-\mathrm{CSFVp})
\end{aligned}
$$

Using the numerical values for $\mathrm{m}$ and CSFVo, Eq. (A3) becomes Eq. (3).

This study was performed under NIA Protocol No. 80-AG-26, entitled "Regional Cerebral Metabolism in Man during Normal Aging". Radiation exposure was within limits set for the normal volunteers by the United States Code of Federal Regulations, Title 21 (Food and Drug Administration, Paragraph 361).

The authors thank the 60 male volunteers for their participation in this study. We also thank Karen Pettigrew for her advice on statistical analysis of the data and Angela Moore for her assistance in recruiting our subjects.

\section{References}

1 Frackowiak RSJ, Gibbs JM. Cerebral metabolism and blood flow in normal and pathological aging. In: Magistretti PL, ed. Functional Radionuclide Imaging of the Brain. New York: Raven Press, 1983:305-9.

2 Frackowiak RSJ, Wise RJS, Gibbs JM, Jones T. Positron emission tomographic studies in aging and cerebrovascular disease at Hammersmith Hospital. Ann Neurol 1984;15(suppl):S1 12-8.

3 Pantano P, Baron J, Lebrun-Grandie P, Duquesnoy N, Bousser P, Comar D. Regional cerebral blood flow and oxygen consumption in human aging. Stroke 1984;15:635-41.

4 Kuhl DE, Metter EJ, Riege WH, Phelps ME. Effects of human aging on patterns of local cerebral glucose utilization determined by the [18F]fluorodeoxyglucose method. J Cereb Blood Flow Metabol 1982;2:163-71.

5 deLeon MJ, George AE, Ferris SH, et al. Positron emission tomography and computed tomography assessment of the aging human brain. $J$ Comput Assist Tomogr 1984;8:88-99.

6 Duara R, Margolin RA, Robertson-Tchabo EA, et al. Cerebral glucose utilization, as measured with positron emission tomography in 21 healthy men between the ages of 21 and 83 years. Brain 1983;106:761-75.

7 Duara R, Grady C, Haxby J, et al. Human brain glucose utilization and cognitive function in relation to age. Ann Neurol 1984;16:702-13.

8 Schwartz M, Creasey H, Grady CL, et al. Computed tomographic analysis of brain morphometrics in $\mathbf{3 0}$ healthy men, aged 21 to 81 years. Ann Neurol 1985;17:146-57.

9 Zatz LM, Jernigan TL, Ahumada AJ. Changes on computed tomography with aging: Intracranial fluid volume. Am J Neuroradiol 1982;3:1-11.

10 Alavi A, Leonard JC, Chawluk J, et al. Correlative studies of the brain with positron emission tomography, nuclear magnetic resonance, and $\mathrm{x}$-ray computed tomography. In: Hartmann A, Hoyer S, eds. Cerebral Blood Flow and Metabolism Measurement. Berlin: Springer-Verlag, 1985:523-39.

11 Herscovitch P, Auchus AP, Gado M, Chi D, Raichle ME. Correction of positron emission tomography data for cerebral atrophy. J Cereb Blood Flow Metabol 1986;6:120-4.

12 Schlageter NL, Horwitz B, Creasey H, Cutler NR, Rapoport SI. Measured gray matter cerebral metabolic rate for glucose and cerebral atrophy are not correlated in a healthy aging population. Neurology 1985;35(suppl 1):232.

13 Shiue C, Salvadori PA, Wolf AP, Fowler JS, MacGregor RR. A new improved synthesis of 2-deoxy-2-[ $\left[{ }^{18} \mathrm{~F}\right]$-fluoro-D-glucose from ${ }^{18} \mathrm{~F}$-labeled acetyl hypofluorite. J Nucl Med 1982;23:899-903.

14 Eycleshymer AC, Schoemaker DM. A Cross-Section Anatomy. New York: D Appleton, 1911:2-55.

15 Huang SC, Phelps ME, Hoffman EJ, Sideris K, Selin CJ, Kuhl DE. Non-invasive determination of local cerebral metabolic rate of glucose in man. Am J Physiol 1980;238:E69-E82.

16 DeLeo JM, Schwartz M, Creasey H, Cutler N, Rapoport SI. Computer-assisted categorization of brain computerized tomography pixels into cerebrospinal fluid, white matter, and gray matter. Comp Biomed Res 1985;18:79-88.

17 DiChiro G, Brooks RA, Dubal L, Chew E. The apical artifact: Attenuation values towards the apex of the skull. J Comput Assist Tomogr 1978;2:65-70.

18 Snedecor GW, Cochran WG. Statistical Methods. 7th Ed. Ames, IA: Iowa State Univ Press, 1980.

19 Mazziotta JC, Phelps ME, Plummer D, Kuhl DE. Quantitation in positron emission computed tomography: 5. Physical-anatomical effects. J Comput Assist Tomogr 1981;5:734-43.

20 Patlak CS, Fenstermacher JD. Measurements of dog blood-brain transfer constants by ventriculocisternal perfusion. Am J Physiol 1975;229:877-84.

21 Johns HE, Cunningham JR. The Physics of Radiology, 4th Ed. Springfield, IL: Charles C. Thomas, 1983.

22 Duara R, Barker W, Chang J, Apicella A, Finn R, Gilson A. Age and sex differences in cerebral glucose consumption measured by PET using $\left[{ }^{18} \mathrm{~F}\right]$-fluorodeoxyglucose (FDG). J Nucl Med 1985;26:P68.

23 Horwitz B, Duara R, Rapoport SI. Age differences in intercorrelations between regional cerebral metabolic rates for glucose. Ann Neurol 1986;19:60-7.

24 Arai H, Kobayashi K, Iked K, Nagao Y, Ogihara R, Kosaka K. A computed tomography study of Alzheimer's disease. $J$ Neurol Sci 1983;229:69-77.

25 Brinkman SD, Sarwar M, Levin HS, Morris HH. Quantitative indexes of computed tomography in dementia and normal aging. Radiology 1981;138:89-92.

26 Creasey H, Schwartz M, Fredrickson H, Haxby J, Cutler NR, Rapoport SI. Brain morphometrics in Alzheimer's disease: Quantitative computed tomographic analysis. Arch Neurol 1986; (in press). 